\title{
Szemerédi Receives 2012 Abel Prize
}

The Norwegian Academy of Science and Letters has awarded the Abel Prize for 2012 to ENDRE SzEMERÉDI of the Alfréd Rényi Institute of Mathematics, Hungarian Academy of Sciences, and

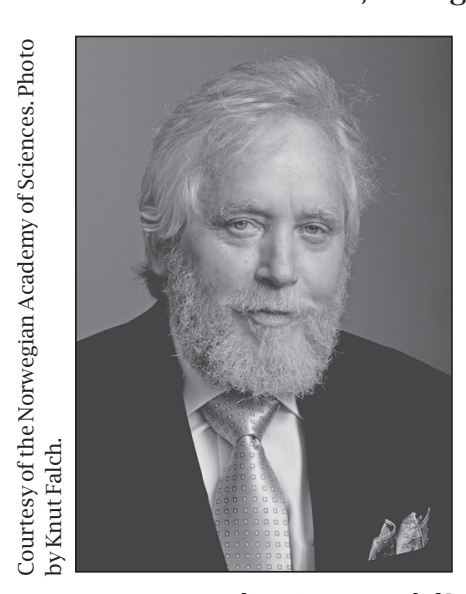

Rutgers University "for his fundamental contributions to discrete mathematics and theoretical computer science, and in recognition of the profound and lasting impact of these contributions on additive number theory and ergodic theory." The Abel Prize recognizes contributions of extraordinary depth and influence to the mathematical sciences and has been awarded annually since 2003. It carries a cash award of 6,000,000 Norwegian kroner (approximately US $\$ 1$ million). Endre Endre Szemerédi Szemerédi received the Abel Prize at an award ceremony in Oslo, Norway, on May 22, 2012.

\section{Citation}

Discrete mathematics is the study of structures such as graphs, sequences, permutations, and geometric configurations. The mathematics of such structures forms the foundation of theoretical computer science and information theory. For instance, communication networks such as the Internet can be described and analyzed using the tools of graph theory, and the design of efficient computational algorithms relies crucially on insights from discrete mathematics. The combinatorics of discrete structures is also a major component of many areas of pure mathematics, including number theory, probability, algebra, geometry, and analysis.

Endre Szemerédi has revolutionized discrete mathematics by introducing ingenious and novel techniques and by solving many fundamental problems. His work has brought combinatorics to the center stage of mathematics by revealing its deep connections to such fields as additive

DOI: http://dx.doi.org/10.1090/noti855 number theory, ergodic theory, theoretical computer science, and incidence geometry.

In 1975 Endre Szemerédi first attracted the attention of many mathematicians with his solution of the famous Erdős-Turán conjecture, showing that in any set of integers with positive density, there are arbitrarily long arithmetic progressions. This was a surprise, since even the case of progressions of lengths 3 or 4 had earlier required substantial effort by Klaus Roth and by Szemerédi himself, respectively.

A bigger surprise lay ahead. Szemerédi's proof was a masterpiece of combinatorial reasoning and was immediately recognized to be of exceptional depth and importance. A key step in the proof, now known as the Szemerédi Regularity Lemma, is a structural classification of large graphs. Over time, this lemma has become a central tool of both graph theory and theoretical computer science, leading to the solution of major problems in property testing and giving rise to the theory of graph limits.

Still other surprises lay in wait. Beyond its impact on discrete mathematics and additive number theory, Szemerédi's theorem inspired Hillel Furstenberg to develop ergodic theory in new directions. Furstenberg gave a new proof of Szemerédi's theorem by establishing the multiple recurrence theorem in ergodic theory, thereby unexpectedly linking questions in discrete mathematics to the theory of dynamical systems. This fundamental connection led to many further developments, such as the Green-Tao theorem asserting that there are arbitrarily long arithmetic progressions of prime numbers.

Szemerédi has made many additional deep, important, and influential contributions to both discrete mathematics and theoretical computer science. Examples in discrete mathematics include the Szemerédi-Trotter theorem, the AjtaiKomlós-Szemerédi semirandom method, the Erdős-Szemerédi sum-product theorem, and the Balog-Szemerédi-Gowers lemma. Examples in theoretical computer science include the AjtaiKomlós-Szemerédi sorting network, the FredmanKomlós-Szemerédi hashing scheme, and the PaulPippenger-Szemerédi-Trotter theorem separating deterministic and nondeterministic linear time. Szemerédi's approach to mathematics exemplifies 
the strong Hungarian problem-solving tradition. Yet the theoretical impact of his work has been a game changer.

\section{Biographical Sketch}

Endre Szemerédi was born in Budapest, Hungary, in 1940. He received his Ph.D. from Moscow State University in 1970 under the direction of Israel M. Gelfand. He has held visiting positions at Stanford University (1974), McGill University (1980), the University of South Carolina (1981-1983), and the University of Chicago (1985-1986). He was the Fairchild Distinguished Scholar at the California Institute of Technology in 1987-1988. He is also a recipient of the Aisenstadt Chair at the Centre de Recherches Mathématiques, University of Montreal. In 2008 he was the Eisenbud Professor at the Mathematical Sciences Research Institute, Berkeley. He has received many awards and honors for his contributions to mathematics and computer science. In 2008 he was awarded the Leroy P. Steele Prize for Seminal Contribution to Research by the AMS. In the same year he received the Rolf Schock Prize in Mathematics from the Royal Swedish Academy of Sciences. Other prizes include the Grünwald Prize (1967, 1968), the Rényi Prize (1973), the Pólya
Prize for Achievement in Applied Mathematics of the Society for Industrial and Applied Mathematics (SIAM) (1975), and the Prize of the Hungarian Academy of Sciences (1979). He has been a member of the Hungarian Academy of Sciences since 1987 and of the U.S. National Academy of Sciences since 2010.

\section{About the Prize}

The Niels Henrik Abel Memorial Fund was established in 2002 to award the Abel Prize for outstanding scientific work in the field of mathematics. The prize is awarded by the Norwegian Academy of Science and Letters, and the choice of Abel Laureate is based on the recommendation of the Abel Committee, which consists of five internationally recognized mathematicians.

Previous recipients of the Abel Prize are: JeanPierre Serre (2003), Michael Atiyah and I. M. Singer (2004), Peter Lax (2005), Lennart Carleson (2006), S. R. S. Varadhan (2007), John G. Thompson and Jacques Tits (2008), Mikhail L. Gromov (2009), John Tate (2010), and John Milnor (2011).

- From announcements of the Norwegian Academy of Science and Letters

\section{Pearl Receives 2011 Turing Award}

The Association for Computing Machinery (ACM) has announced that JUDEA PEARL of the University of California, Los Angeles, has been named the recipient of the 2011 ACM A. M. Turing Award "for fundamental contributions to artificial intelligence through the development of a calculus for probabilistic and causal reasoning." The award carries a cash prize of US\$250,000 and will be presented to Pearl at the annual ACM Awards Banquet on June 16, 2012, in San Francisco, California.

\section{Citation}

Judea Pearl's work has transformed artificial intelligence (AI) by creating a representational and computational foundation for the processing of information under uncertainty. Pearl's work went beyond both the logic-based theoretical orientation of AI and its rule-based technology for expert systems. He identified uncertainty as a core problem faced by intelligent systems and developed an algorithmic interpretation of probability theory as

DOI: http://dx.doi.org/10.1090/noti854 an effective foundation for the representation and acquisition of knowledge.

Focusing on conditional independence as an organizing principle for capturing structural aspects of probability distributions, Pearl showed how graph theory can be used to characterize conditional independence and invented message-passing algorithms that exploit graphical structure to perform probabilistic reasoning effectively. This breakthrough has had major impact on a wide variety of fields where the restriction to simplified models had severely limited the scope of probabilistic methods; examples include natural language process-

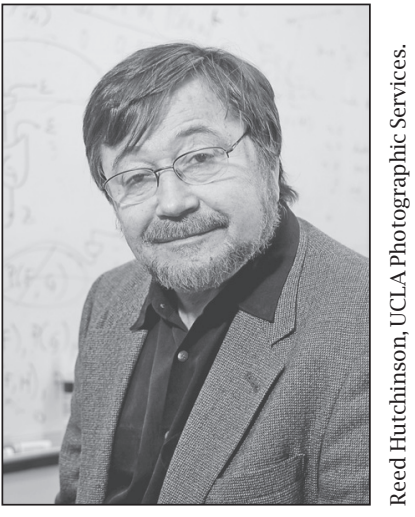

Judea Pearl ing, speech processing, computer vision, robotics, computational biology, and error-control coding.

Equally significant is Pearl's work on causal reasoning, where he developed a graph-based calculus of interventions that makes it possible to derive 
EH

Eidgenössische Technische Hochschule Zürich

Swiss Federal Institute of Technology Zurich

\section{Professor of Applied} Mathematics

The Department of Mathematics (www.math.ethz.ch) at ETH Zurich invites applications for a position in Applied Mathematics at the Full or Associate Professor level. The vacant position is within the Seminar for Applied Mathematics, SAM (www.sam.math.ethz.ch).

The successful candidate's mathematical results should have received wide international recognition. His or her results should be landmark contributions to mathematical modelling and/or efficient numerical simulation in engineering and the sciences. A strong algorithmic and computational component in his/her mathematical research is expected. The candidate should have demonstrated proficiency in conducting pioneering projects in applied mathematics. Together with other members of the Department, the new professor will be responsible for teaching undergraduate courses (German or English) and graduate courses (English) for students in Applied Mathematics and Computational Science and Engineering (CSE).

Please apply online at www.facultyaffairs.ethz.ch. Your application should include your curriculum vitae and a list of publications. The letter of application should be addressed to the President of ETH Zurich, Prof. Dr. Ralph Eichler. The closing date for applications is 31 July 2012. ETH Zurich is an equal opportunity and affirmative action employer. In order to increase the number of women in leading academic positions, we specifically encourage women to apply. ETH Zurich is further responsive to the needs of dual career couples and qualifies as a family friendly employer. causal knowledge from the combined effects of actions and observations. This work has been transformative within AI and computer science and has had major impact on allied disciplines of economics, philosophy, psychology, sociology, and statistics.

\section{Biographical Sketch}

Judea Pearl is a professor of computer science at the University of California, Los Angeles, where he was director of the Cognitive Systems Laboratory. Before joining UCLA in 1970, he was at RCA Research Laboratories working on superconductive parametric and storage devices. Previously, he was engaged in advanced memory systems at Electronic Memories, Inc. Pearl is a graduate of the Technion, the Israel Institute of Technology, with a B.S. degree in electrical engineering. In 1965 he received a master's degree in physics from Rutgers University and in the same year was awarded a Ph.D. degree in electrical engineering from the Polytechnic Institute of Brooklyn.

Among his many awards, Pearl is the recipient of the 2012 Harvey Prize in Science and Technology from the Technion and the 2008 Benjamin Franklin Medal in Computers and Cognitive Science from the Franklin Institute. He was presented with the 2003 Allen Newell Award from ACM and the AAAI (Association for the Advancement of Artificial Intelligence). His groundbreaking book on causality, Causality: Models, Reasoning, and Inference, won the 2001 Lakatos Award from the London School of Economics and Political Science "for an outstanding significant contribution to the philosophy of science."

Pearl is a member of the National Academy of Engineering and a Fellow of AAAI and the Institute for Electrical and Electronic Engineers (IEEE). He is president of the Daniel Pearl Foundation, named after his son.

\section{About the Award}

The A. M. Turing Award was named for Alan M. Turing, the British mathematician who articulated the mathematical foundation and limits of computing and who was a key contributor to the Allied cryptanalysis of the German Enigma cipher during World War II. Since its inception in 1966, the Turing Award has honored the computer scientists and engineers who created the systems and underlying theoretical foundations that have propelled the information technology industry.

The award is given to an individual selected for contributions of a technical nature made to the computing community. The contributions should be of lasting and major technical importance to the computer field. Financial support for the Turing Award is provided by the Intel Corporation and Google Inc.

- ACM announcement 